\title{
Las Narraciones de Experiencias Personales en la Escuela Infantil ¿Cómo Apoyan las Maestras la Participación de los Niños?
}

\section{Children Telling Personal Experiences in Kindergarten. How do Teachers Promote Children's Participation?}

\author{
Celia Renata Rosemberg y María Soledad Manrique \\ CONICET
}

\begin{abstract}
El trabajo se propone analizar cualitativamente situaciones de relato de experiencias personales en el evento de la ronda en la escuela infantil, atendiendo en particular a las estrategias que las maestras emplean para colaborar con los niños en la reconstrucción de su experiencia a través de una narración bien estructurada. Empleando el método comparativo constante (Glasser \& Strauss, 1967) para analizar 98 situaciones videofilmadas, se identificaron y caracterizaron los mecanismos discursivos subyacentes tanto a las situaciones en las que las maestras se apoyan en las pistas proporcionadas por los niños para interpretar su intención comunicativa, como a aquellos en que las maestras se alejan de la intención comunicativa de los niños y centran el intercambio en aspectos periféricos del discurso infantil.
\end{abstract}

Palabras Clave: interacción en el aula, relato de experiencias personales, escuela infantil, estrategias docentes.

\begin{abstract}
The aim of this study is to qualitatively analize situations in which children tell personal experiences in the circle at kindergarten. We focus on how teachers scaffold children as they tell personal experiences through a good structured narrative. We analysed 98 situations of collaborative retelling using the constant comparative method (Glasser y Strauss, 1967). In some cases teachers interpret the child's intention through certain cues in the child's discourse and assist the child as he/she verbally reconstructs his/her experience. In other situations teachers diverge from the child's intention and focus verbal interaction in topics which are periferic to the child discourse.
\end{abstract}

Keywords: classroom interaction, narrative of personal experience, kindergarten, teacher's strategies.

En la mayoría de las escuelas infantiles americanas y europeas ${ }^{1}$ las actividades cotidianas comienzan con una rutina específica destinada a que los niños intercambien sobre algún tema o experiencia de su interés, poniendo en juego sus recursos lingüísticos para producir principalmente discurso narrativo. Esta situación se denomina la ronda por las características espaciales que adopta: los niños se ubican en círculo junto con la maestra para escuchar los relatos e intercambiar en torno a ellos.

Celia Renata Rosemberg, CIIPME (Centro Interdisciplinario de Investigación en Psicología y Matemática Experimental), dependiente del CONICET, Argentina. María Soledad Manrique, Becaria CIIPME (Centro Interdisciplinario de Investigación en Psicología y Matemática Experimental), dependiente del CONICET, Argentina. La correspondencia relativa a este artículo deberá dirigirse a las autoras: Av. Fleming 949, Dpto. B, Martínez, Provincia de Buenos Aires, Argentina. E-mail: solemanrique@conicet.com.ar; crrosem@hotmail.com

1 Diversos trabajos realizados en EE.UU y Europa documentan esta práctica (Cazden, 1988; Green, Kantor \& Rotger, 1991; Mendez \& Lacasa, 1997; Michaels, 1988; Ochs \& Capps, 1996; Póveda, Sebastián \& Moreno, 2003; Snow, 1983).
Desde diversas perspectivas, se ha considerado que la ronda constituye una situación clave en tanto cumple funciones sociales, emocionales, lingüísticas y cognitivas. Así, por ejemplo, se ha mostrado que esta situación, al contribuir al desarrollo de las habilidades de participación de los niños (Green, Kantor \& Rotger, 1991) y al integrar experiencias extraescolares al entorno escolar (Mendez \& Lacasa, 1997), facilita los procesos de socialización en la escuela infantil. En el proceso de participación que tiene lugar en la ronda los niños presentan a la maestra y a sus compañeros una imagen de sí mismos y al hacerlo reafirman su propia identidad (Ochs \& Capps, 1996). La participación conjunta da lugar a la construcción de significados comunes entre la maestra y los niños (Feldman, 1999 citado en Póveda, Sebastián \& Moreno, 2003), al desarrollo de una historia grupal compartida (Green, Kantor \& Rotger, 1991; Póveda, 2003) y, por lo tanto, a la constitución de una identidad grupal. La necesidad de reelaborar discursivamente la experiencia para poder comunicarla a otros promueve el desarrollo de las habilidades discursivas y comunicativas de los 
niños (Cazden, 1989), en particular, el formato de discurso narrativo (Michaels, 1991) y un estilo descontextualizado de lenguaje (Michaels, 1988; Snow, 1983).

Aun cuando se trata de un evento colaborativo, en el que cada uno de los relatos está mediatizado por las interpretaciones, las expectativas y los aportes de todos los participantes que conforman el grupo (Póveda, 2003), la maestra ocupa un lugar muy importante. Es ella quien organiza y coordina el evento, y la calidad de sus estrategias afectará el desarrollo en los niños de los aspectos lingüísticos sociales y afectivos mencionados. En el presente trabajo, el análisis de la interacción durante el relato de experiencias personales en la ronda se focaliza, precisamente, en las estrategias de las maestras para colaborar con los niños en la reelaboración lingüística de su experiencia pasada.

\section{Encuadre Teórico}

La narración de experiencias personales constituye una forma de recapitular la experiencia pasada, que implica el establecimiento de una correspondencia entre una secuencia de cláusulas verbales y una secuencia de eventos que de hecho tuvieron lugar y que luego pueden inferirse de esas cláusulas (Labov, 1972). El hablante organiza estas cláusulas en una estructura conformada por ciertas unidades: un resumen, una orientación en el tiempo y el espacio, una complicación de la acción, una resolución del conflicto planteado, una coda y una o varias evaluaciones (Lavob, 1972).

Desde una perspectiva evolutiva, las narraciones constituyen el primer género de discurso en desarrollarse. Ya entre los 2 y los 2:6 años los niños pueden producir una diversidad de narrativas (Miller, 1994; Miller \& Sperry, 1987; Nelson, 1991; Preece, 1987). En efecto, como parte del proceso de socialización, a través de su participación en eventos en los que esta forma discursiva se pone en juego, los niños van internalizando el esquema implícito en las narrativas así como también las estrategias y las fórmulas de señalización (Gumperz, 1984) que este formato tiene en su cultura.

Las condiciones de desarrollo de este género discursivo están dadas por ciertas habilidades cognitivas, esto es, por la capacidad de representarse la experiencia en una secuencia temporal (Bruner, 1986; Nelson, 1991), por el sostén que proporciona la interacción con otras personas -sostén que constituye la matriz en la que se producen las primeras narraciones (Miller, 1994; Miller \& Sperry, 1987;
Nelson, 1991)-y por el significado emocional que el evento relatado tiene para el niño (Fivush \& Haden, 1997). La focalización particular que el narrador imprime a su experiencia conlleva la interpretación implícita o explícita de las acciones en términos de intenciones, motivaciones, afectos, creencias o valores (Bruner, 1986, 1988).

Los aspectos cognitivos, emocionales e interactivos mencionados han sido analizados en detalle en investigaciones sobre las conversaciones madre-niño en torno a eventos pasados (Nelson, 1996; Reese, Haden \& Fivush, 1993). Estos trabajos mostraron el impacto del lenguaje en el pensamiento, en especial en la configuración de representaciones mentales narrativas, sobre las que se estructuran la memoria episódica y autobiográfica que son la base para la constitución de la propia identidad (Nelson, 1996).

Estas investigaciones, asimismo, señalaron el papel de la interacción social en el desarrollo del discurso narrativo. Así, por ejemplo, el trabajo de Engel (1986 citado en Nelson, 1996) mostró que, mientras algunas madres en la interacción proporcionan un hilo narrativo que conforma un marco para la participación de los niños en el evento (estilo narrativo), otras madres se focalizan en información aislada sobre la acción y los participantes (estilo repetitivo o pragmático). En el mismo sentido, los estudios cuasi-experimentales de Tessler y Nelson (1994) describieron la textura conversacional, narrativa o pragmática, construida por la madre en torno a un evento del que participa con su hijo y analizaron las diferencias entre niños en el recuerdo del evento. Los resultados de estos trabajos revelaron que los niños cuyas madres presentan un estilo de interacción narrativo no sólo recuerdan los eventos pasados en mayor medida, sino que además suelen estructurarlos de modo coherente en un formato narrativo.

Rosemberg y Borzone de Manrique (1994), en su análisis de episodios de lectura de cuentos en la escuela infantil, encontraron que las maestras, al igual que las madres en los estudios mencionados, se diferencian en función de su estilo de interacción. En efecto, mientras que algunas maestras se centran por medio de preguntas cerradas en la recuperación de información aislada, otras maestras, en cambio, reconstruyen narrativamente el texto. Estas maestras, así como también las madres que "narrativizan" la experiencia infantil, retoman en su discurso la participación verbal y no verbal del niño en la situación, explicitan las intenciones y las relacionan con las acciones que han tenido lugar. 
Estos procesos de colaboración son posibles sólo cuando el adulto es capaz de inferir la intención comunicativa del niño. Las inferencias, base de la comprensión mutua (Gumperz, 1984), resultan más sencillas para el adulto cuando en el intercambio tiene una referencia compartida con el niño: el texto escrito en el caso de las situaciones de lectura de cuentos en el aula o la experiencia vivida conjuntamente en el relato de experiencias en el medio familiar (Rosemberg \& Manrique, 2004, 2005). Distinto es el caso de las situaciones de relato de experiencias personales en la ronda. Como la maestra generalmente no conoce la experiencia que el niño pretende narrar, puede tener mayores dificultades para inferir la intención comunicativa del niño.

Pero en la interpretación del mensaje infantil los adultos pueden recurrir también a pistas a través de las cuales los niños implícitamente transmiten significados en las negociaciones de la conversación. Las pistas de contextualización (Gumperz, 1984) constituyen características lingüísticas indexicales de contenido operacional establecidas convencionalmente que se aprenden como parte de la socialización lingüística primaria y se emplean sin reflexión conciente. Tanto los fenómenos prosódicos como el cambio de código, dialecto o estilo, la elección entre opciones léxicas o sintácticas o las estructuras de secuenciamiento que permiten jerarquizar la información, pueden constituir pistas que conforman un contexto que orienta a los oyentes hacia una interpretación apropiada de sus emisiones.

Como lo mostró Michaels (1988), cuando el adulto no atiende o no logra interpretar las pistas de contextualización empleadas por el niño, las interacciones no dan lugar a la comprensión mutua. En un estudio etnográfico analiza cómo la interpretación errónea que una maestra realiza de las claves prosódicas empleadas por los niños negros le impide identificar los momentos de transición en los cuales tomar el turno de habla sin interrumpir el relato del niño. Esto resulta en una falta de sincronización en los intercambios y en una evaluación no acertada de la intención comunicativa del otro, se trate tanto de la maestra como del niño con el que está interactuando.

Por su parte, Póveda (1999, 2001, 2003), en el análisis que realiza sobre el relato de experiencias personales de un niño gitano, afirma que las diferencias culturales entre la maestra y los niños no siempre constituyen un impedimento para que tanto la maestra como los niños evalúen positivamente su participación en la ronda. Póveda observa que aún cuando el niño gitano tiende a elaborar relatos no centrados en un tema, empleando una estrategia narrativa de asociación temática, la maestra, al ser conciente de estas diferencias discursivas, puede intervenir proporcionando al niño un andamiaje, sincronizando sus turnos con las pausas que estructuran el relato del niño.

La colaboración que el adulto puede prestar al niño en la producción oral de discurso depende de que éste pueda identificar aquellos aspectos del evento en los que el niño se ha focalizado, así como la perspectiva personal desde la que pretende comunicarlos. La organización de la información en el relato no es arbitraria: la secuencia de emisiones en el discurso infantil constituye una "pista" que permite inferir cómo el niño ha jerarquizado la información. En general, en las lenguas sujeto-verbo-objeto como el español, la información que se focaliza se presenta al final a partir del verbo (Pardo, 1986). El hablante dice primero aquello sobre lo que se sustenta -el tema-y posteriormente predica algo sobre ello -el rema-, aquello que realmente quiere transmitir, aquello que posee mayor carga semántica (Firbas, 1992).

Pardo (1986) reformula a nivel textual las nociones de tema oracional y rema oracional. En el nivel del texto la jerarquización de la información se manifiesta en el orden en que el hablante presenta la información. De ahí que, canónicamente, el tema del texto aparece al comienzo en una "emisión líder", que establece los patrones semánticos y pragmáticos en el marco de los cuales el hablante producirá el texto. Los remas de las emisiones, hacen alusión directa o indirecta al tema del texto y se van sucesivamente tematizando hasta llegar a aquella información más novedosa en términos de la intención comunicativa del hablante: el rema del texto.

Cuando se trata del texto de un niño producido en la interacción con un adulto cabe preguntarse cómo las intervenciones del adulto se "entretejen" con las del niño y resultan en un relato que puede responder en mayor o menor medida a la intención comunicativa que se infiere de las primeras emisiones del niño.

En este marco, el presente trabajo se propone precisamente analizar las interacciones entre las maestras y los niños de la escuela infantil que dan lugar a la elaboración conjunta de narraciones. En el análisis se consideran las estrategias de interacción de las maestras en relación a las emisiones infantiles, atendiendo a si estas estrategias se apoyan en una interpretación de la intención comunicativa del niño y configuran un andamiaje que recupera la in- 
formación que el niño ha jerarquizado en su discurso para colaborar con él en la reconstrucción narrativa de su experiencia.

\section{Método}

\section{Recolección de Información Empírica}

El presente trabajo es parte de una investigación más amplia sobre la interacción maestra-niños/as en escuelas infantiles de poblaciones urbano-marginadas del conurbano de Buenos Aires ${ }^{2}$. Se trata de escuelas con reconocimiento privado de la Provincia de Buenos Aires, pero que se hallan articuladas con asociaciones civiles o religiosas insertas en las comunidades. El personal a cargo de los niños posee título de profesor de nivel inicial. Todos los niños que atienden provienen de hogares en situación de extrema pobreza.

En siete salas de 3, 4 y 5 años de dos centros infantiles se registraron por medio de audio y video (116 horas de grabación) las situaciones frecuentes que componen la rutina escolar. El corpus comprende 114 situaciones: rondas (36), lecturas de cuento (26), situaciones de juego (27), actividades de enseñanza de aspectos específicos de la lectura y la escritura o la matemática (25). Para este trabajo se analizaron los 98 relatos de experiencias personales elaborados por los niños en la interacción con sus maestras en las 36 situaciones de ronda (entre 2 y 3 relatos por ronda).

\section{Procedimiento Para el Análisis de la Información}

Los relatos se analizaron empleando un procedimiento cualitativo que combina la elaboración inductiva de categorías en base al método comparativo constante (Glasser \& Strauss, 1967) y su consideración atendiendo a otros marcos conceptuales. Un análisis preliminar (Rosemberg \& Manrique, 2005), que se realizó sobre un corpus limitado de situaciones de ronda considerando conceptos del análisis del discurso (Pardo, 1986), se complejizó en el presente trabajo atendiendo a conceptos sociolingüísticos (Gumperz, 1984; Labov, 1972).

A partir de un procedimiento conjunto de codificación y análisis (Strauss \& Corbin, 1991; Valles, 2003) se identificaron una serie de dimensiones que permitieron establecer un primer orden en los datos:

1. Quién inicia el intercambio y propone la emisión líder.

2. Cuál es el tema del discurso y cómo el tema se va especificando progresivamente.

3. El grado en el que la maestra retoma el tema propuesto por el niño y los aspectos en los que éste se focaliza.

4. Las estrategias de las maestras que contribuyen a configurar las diversas unidades del relato del niño -resumen, orientación, complicación, resolución, coda y evaluación- (Lavob, 1972).

5. La participación de los niños en relación con las estrategias de las maestras.

En los 98 relatos se identificó cómo se combinaban típicamente las categorías mencionadas y se elaboró una

2 “Conversación y desarrollo lingüístico en la infancia. Un estudio de la interacción verbal en el jardín de infantes con población urbano-marginal". Tesis doctoral en preparación de Manrique, M. S. (2004-2005). serie de categorías o "tipos" de situaciones de relato compartido que permitía dar cuenta del corpus completo. Estos tipos son:

1. El niño propone el tema, y el andamiaje de la maestra da lugar a la elaboración de un relato bien estructurado que incluye resumen, orientación, complicación, resolución, coda y evaluación y que responde a la jerarquización de la información en el discurso infantil.

2. La maestra plantea el tema pero colabora con el niño para que éste elabore un relato personal, focalizando los aspectos que a él le interesan.

3. La maestra plantea el tema, y sus posteriores intervenciones dirigen la elaboración del relato de acuerdo a su perspectiva del evento y no a la del niño.

4. El niño plantea un tema pero la maestra no le permite desarrollar el relato.

5. El niño plantea el tema pero la maestra en sus intervenciones desplaza el foco y reorienta la elaboración del relato atendiendo a aspectos de su interés.

En los resultados se muestra cómo estos "tipos" se despliegan en la interacción, ilustrando cada uno de ellos con el análisis de casos ejemplares. La forma de presentación responde a las características metodológicas de los estudios comprensivos, que intentan acercar al lector la realidad concreta que conoció el investigador. El análisis conlleva la elaboración de los datos respetando su inserción original en un contexto complejo y la presentación de los rasgos más salientes en una descripción que reproduce para el lector su cualidad singular (Geertz, 1973; Gibaja, 1988). De este modo, por medio del análisis de ejemplos, de transcripciones de episodios y de citas textuales, se intenta una aproximación al ideal de descripción densa, que Geertz (1973) elevó a paradigma del modelo.

\section{Resultados}

El análisis de los relatos de experiencias personales en la ronda mostró diferencias en el grado en el que los textos producidos con la colaboración de la maestra respondían a la intención comunicativa inicial de los niños. El análisis de los datos permitió asimismo identificar las estrategias de las maestras que conforman un andamiaje para que los niños construyan un relato. Mientras que en algunas situaciones las maestras desvían el relato en una dirección que no responde a la intención comunicativa del niño, en otras situaciones el andamiaje de las maestras da lugar a que los niños desplieguen su intención comunicativa por medio de un relato que reconstruye no sólo los sucesos vividos sino también la perspectiva personal que los niños tienen en relación con ellos.

En las situaciones en las que las maestras sintonizan sus intervenciones con las de los niños, estratégicamente retoman la emisión líder del relato y cooperan con ellos en la elaboración de un texto que, en muchos casos, adopta una estructura narrativa desarrollada. Esto se observa, incluso, en los intercambios en los que los niños ponen de manifiesto muchas limitaciones para relatar su experiencia. 
En el ejemplo que se presenta a continuación, las dificultades de Karen para contar en detalle su visita al dentista se deben probablemente a que la niña no ha podido construir durante el evento una representación completa del mismo. En efecto, en estas situaciones no resulta posible registrar visualmente la secuencia de acciones que realiza el dentista (o al menos aquellas operaciones que tienen lugar dentro de la boca). A estas acciones se puede acceder únicamente de modo indirecto si el dentista las verbaliza a medida que las lleva a cabo, o si el niño ha construido, a partir de experiencias previas vicarias (T.V., otros relatos, etc.), una representación mental rutinizada -un guión- de lo que sucede cuando uno va al dentista, y a partir de ese guión puede inferir la información que no registró de modo directo. De ahí que la maestra recurra a diversas fuentes de conocimiento para cooperar con la niña en la reconstrucción lingüística del evento.

(1) Karen (4:1 años)

Maestra: Dale, va a contar Karen.

Karen: Fui al dentista.

Maestra: ¿Fuiste al dentista? ¿Y qué pasó? Contame ¿Porqué fuiste al dentista?...

Karen: Me dolía la muela.

Maestra: ¿Te dolía la muela? ¿Y qué pasó?... ¿Qué te dijo el dentista cuando te vio?

Karen: Dijo que me... que... (habla muy bajo)

Maestra: ¿Qué hizo?

Karen: ...que no coma.

Maestra: ¿Por qué te dijo que no comas de ese lado, qué te hizo?

Karen: Porque me duele la muela mucho.

Maestra: Ahí va. Ella está diciendo algo. Karen estuvo contando, Elian y Melanie (que conversaban), que fue al dentista porque su muela le dolía ¿Te la arregló?

(Karen asiente)

Maestra: ¿Sí? ¿Cómo te la arregló?

Kevin: ¡Te la sacó!

Maestra: ¿Te sacó la muela, dice Kevin?

Karen: No.

M: No le sacó la muela ¿Qué te hizo?

(Karen se toca la boca y no responde)

...

Maestra: (Niega con la cabeza) ¿Te la sacó o te la arregló?

Karen: Me la arregló.

Maestra: Te la arregló porque, ¿Qué tenía ella acá?

(Karen no responde)

Silvana: Le puso pasta.
Maestra: Le puso pasta porque seguramente su muela tenía una caries, tenía un agujerito. Entonces... le puso pasta. ¿Y después el dentista qué le dijo? ¿A ver?

$\cdots$

Maestra: ...Lo que pasa es que los dentistas cuando tenemos dolor de muelas y tenemos un agujerito que es una carie, no nos sacan las muelas. Nos las arreglan. Y me parece que tiene razón Silvana que no se la sacaron, que le pusieron pasta. Mami si no me hubiera dicho que le sacaron la muela; Y mami nunca me dijo que le sacaron la muela! Te la arreglaron y por eso el dentista te dijo que no comieras de ese lado. Porque qué pasa, Silvana, ¿Vos sabés, no?

Silvana: Te duele la muela.

Maestra: Después de que nos ponen esa pastita no podemos comer de ese lado.

Maestra: Entonces, Karen, ¿qué pasó después de que el médico te dijo que no comieras de ese lado?

Karen: Me puso pasta.

En el intercambio, la maestra retoma la emisión líder de la niña -Fui al dentista- y a partir de este anclaje intenta que expanda la complicación del evento a través de una pregunta abierta que no imprime una dirección determinada al relato de la niña - ¿Fuiste al dentista? ¿Y qué pasó? Contame, ¿Porqué fuiste al dentista? ... ¿Qué pasaba?-. Karen proporciona una respuesta muy general. De ahí que las siguientes intervenciones de la maestra estén destinadas a que la niña detalle los eventos que tuvieron lugar en la visita al dentista - ¿Qué te dijo el dentista cuando te vio? ¿Qué hizo?... ¿Por qué te dijo que no comas de ese lado, qué te hizo?-.

Ante las dificultades de Karen para especificar la información, la maestra ofrece alternativas de respuestas - ¿Te la arregló?... ¿Cómo te la arregló? ¿Te la sacó o te la arregló?-y retoma también alternativas de respuesta que ofrecen otros niños-Kevin: ¡Te la sacó! ... Maestra: ¿Te sacó la muela, dice Kevin?-. La maestra se vale de sus propias representaciones sobre la situación para completar la información ausente en la secuencia de la complicación del evento y para establecer relaciones con la información que Karen proporciona-Le puso pasta porque seguramente su muela tenía una caries, tenía un agujerito. Entonces... le puso pasta y después el dentista ¿qué le dijo? ¿A ver?-. 
El relato que se construye en la interacción representa una versión lingüística de los hechos apoyada en la información que proporciona la niña y en otras fuentes de conocimiento general. Cabe señalar que la maestra intenta justificar su "versión" a través de distintas estrategias. Presenta evidencias que sustentan su reconstrucción particular de los hechos-Mami si no me hubiera dicho que le sacaron la muela; Y mami nunca me dijo que le sacaron la muela!-, y explicita aquello que sucede habitualmente en las visitas al dentista-Lo que pasa es que los dentistas cuando tenemos dolor de muelas y tenemos un agujerito que es una carie, no nos sacan las muelas. Nos las arreglan- señalando que esa visita particular constituye un caso del guión "ir al dentista".

La maestra no esconde el carácter de interpretación que tiene la reconstrucción que hace de la experiencia de la niña, hecho que se pone de manifiesto en el intercambio, a través del uso de modalizadores $-Y$ me parece que tiene razón Silvana que no se la sacaron, que le pusieron pasta-. Estos indicadores constituyen pistas que permiten a los niños inferir que el relato elaborado en la interacción puede diferir en alguna medida de las acciones que efectivamente realizó el dentista con Karen. De este modo, la maestra implícitamente transmite a los niños que siempre existen diferencias más o menos sutiles entre los hechos reales y la reconstrucción lingüística de estos hechos.

En otras situaciones no es el niño sino la maestra quien propone el tema para un relato compartido. En estos casos las maestras eligen tópicos que suponen interesantes para los niños, o bien porque los han escuchado comentar sobre ellos o porque son eventos muy relevantes en la vida de los niños (nacimientos, mascotas, mudanzas y accidentes, cumpleaños y paseos, etc.). En algunas de estas situaciones, aún cuando es la maestra quien plantea la emisión líder del texto, proporciona un amplio espacio de participación que permite que los niños orienten el relato, contribuyendo con especificaciones remáticas del tema central. Por ejemplo, en el siguiente fragmento la maestra propone conversar sobre el nacimiento del bebé de otra maestra, tema sobre el que la maestra había escuchado una conversación espontánea entre los niños. En los sucesivos turnos, las contribuciones de los niños se focalizan en diversos aspectos de su interés.

(2) Ezequiel (3:8 años), Cristian (4:2 años)

Maestra: Ah, pueden contar lo que estaban con- tando ahi en la mesa. Que la señorita Silvina tuvo un bebé.

Niño: Tuvo un bebé.

Ezequiel:...pero era una pelota.

Maestra: ...¿Quién es una pelota?

Ezequiel: El bebito.

Cristian: Era gorda... (no se le entiende)

Maestra: Estaba gorda.

...

Maestra: ... ¿Cómo estaba, dijiste vos Cristian?

Ezequiel: Tenía gorda.

Cristian: Tenía gorda... después tenía una pelotita.

Maestra: Paren ¿Tenía gorda o estaba gorda? ¿Cómo se dice?

Cristian: Estaba gorda, estaba gorda.

Maestra: Estaba muy gorda, ¿por qué?

Ezequiel: Porque tenía un bebé...

Maestra: Y cómo se dice cuando una... ¿Cómo se dice cuando una señora... tiene un bebé adentro de la panza, que está qué? Como la mamá de Aixa ¿Cómo está tu mamá, está...?

Ezequiel: Gorda.

Aixa: Embarazada.

Maestra: Embarazada. Estaba muy gorda porque estaba embarazada.

Al comenzar el intercambio los niños se centran en las transformaciones físicas que el embarazo conlleva para la madre-era una pelota-. Las intervenciones de la maestra en este fragmento de intercambio tematizan esta afirmación inicial de uno de los niños $\mathrm{y}$, a través de aportes sucesivos, la reconceptualizan precisando el concepto -Era una pelotal Era gorda/ Estaba gorda/ Estaba gorda, ¿Por qué?/ Porque tenía un bebé- hasta llegar al término apropiado: Embarazada.

En otros episodios, la maestra además de proponer el tema imprime una focalización precisa sobre aquellos aspectos en los que le interesa que los niños se centren. Esto es lo que sucede en el siguiente fragmento en el que la maestra propone a una niña que relate la mudanza de su familia y orienta el desarrollo del texto a través de preguntas cerradas.

(3) Tatiana (4:3 años)

Maestra: Tatiana tiene una novedad ¿Qué hiciste Tati este fin de semana? Algo re importante que pasó en tu casa.

Tatiana: Que me cambié de casa.

Maestra: Te cambiaste de casa. Se mudó y ¿A dónde te mudaste?

Tatiana: A la vuelta de la escuela. 
Maestra: Acá, a la vuelta de la escuelita... ¿Y cómo es la casa nueva?, contanos. Porque yo la casa de antes la conocía que era en el pasillo.

Tatiana: Es una casa blanca y tiene una... (gesto de zigzag).

Maestra: Tiene una puerta blanca con un vidrio y una reja, ¿Una reja finita?

(Tatiana niega con la cabeza)

Maestra: Una rejilla.

(Tatiana asiente)

Maestra: ¿Y después?

Tatiana: Y tiene patio.

Maestra: ¿Tiene patio? Y tu mamá me dijo algo re importante que tiene en el patio, algo re lindo que tiene en el patio. ¿No tenés un árbol en el patio?

(Tatiana asiente)

Maestra: O sea que es un patio grande, jestá bueno!... ¿Y ya mudaron todo Tatiana ofalta algo?

(Tatiana asiente)

Niño: Yo tenía una pileta.

Maestra: Tenés una pileta, porque tenés un patio grande, la ponés en el patio.

Como se observa en el intercambio, la emisión inicial de la maestra no sólo le indica a Tatiana a través de una pregunta el tema del relato, sino que también constituye una evaluación del evento - algo re importante-. Así, la maestra jerarquiza la información que le pide a la niña que proporcione, jerarquía que afectará la forma en que la niña presente la información, así como también la atención que los otros niños presten al relato.

Es interesante mencionar que en el proceso de reconceptualización que la maestra realiza de la respuesta de Tatiana-me cambié de casa-, modifica el interlocutor de la emisión: la maestra se dirige primero a la niña, repitiendo su emisión - te cambiaste de casay cuando se dirige a todo el grupo reconceptualiza la emisión infantil, precisando el concepto -se mudó-. Luego incita a la niña a conservar su turno por medio de preguntas referidas en su mayoría a aspectos descriptivos más que a secuencias de acciones, propias del género narrativo. Las preguntas cerradas que realiza la maestra-¿Una reja finita?/¿Yya mudaron todo Tati o falta algo?- así como sus comentarios evaluativos respecto de la información que ya posee -Y tu mamá me dijo algo re importante que tiene en el patio, algo relindo que tiene en el patio ¿No tenés un árbol en el patio?- jerarquizan la información y orientan las intervenciones de la niña hacia los aspectos que resultan de su interés.
Al final del fragmento, cuando un niño participa de un intercambio con un comentario que parece iniciar un nuevo relato -Yo tenía una pileta- la maestra sin permitirle desarrollar un nuevo relato, liga esa emisión al relato previo -Tenés una pileta, porque tenés un patio grande, la ponés en el patio-.

Son frecuentes los casos extremos en que las maestras cortan los relatos de los niños sin permitirles desplegar su intención comunicativa en un texto extenso. Mientras que en algunas situaciones las maestras relacionan la iniciación del niño con el relato previo, como se observó en el caso anterior, en otras situaciones interrumpen la narración de un niño simplemente para otorgarle el turno a otro, como se muestra en el siguiente fragmento.

(4) Keila (5:1 años)

Keila: Que... que había un piso alto; yo estaba caminando en el piso chiquito y me caí.

Maestra: Sí, ya nos contaste (tono burlón); ¿Cuando fuiste a comprar al kiosco de tu casa?

(Keila asiente)

Maestra: Sí, bueno, ya está ¿Qué me contabas Sabrina?

La tendencia de las maestras a orientar los relatos de los niños en una dirección particular resulta muy evidente en las situaciones en las que las maestras, aun cuando parecen otorgar al niño un espacio para que reconstruya su experiencia personal, por medio de sus intervenciones, reorientan el relato del niño. De este modo, desplazan el foco de la información en la que el niño se había centrado a información que en el relato infantil aparecía en forma periférica.

(5) Brian (4:2 años)

Brian: Seño, ¿Sabes qué? El otro día yo fui a la casa del trabajo, un departamento chiquito con mi mamá y fui a abrir la puerta y salió un perro. No, primero estaba en el departamento tomando jugo, galletitas y mi mamá me dio... fue y había un perro que era muy grandote, que era grandote y fue al patio y salió el perro por la escalera, yo estaba en la escalera y el perro... salió.

Maestra: ¿Vos acompañaste a tu mamá al trabajo? Brian: Sí.

...

Maestra: ¿Tu mamá trabaja en una casa?

Brian: En un departamento.

Maestra: Es diferente en un departamento. 


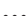

Maestra: Y vos fuiste con tu mamá, ¿Tu mamá limpia el departamento?

Brian: Si y había un perro y casi me muerde.

Maestra: ¿Y en el departamento tenía escalera para subir o...? ¿... ¿Había timbre, tenía timbre el departamento?

Brian: Sí.

Maestra: Mirá que bueno, esas mamás que limpian las casas ¿Alguna otra mamá limpia, como la mamá de Brian?

Brian: Tenía una boca re grande.

Maestra: ¿Quién?

Brian: El perro.

Maestra: Bueno, seguimos con la historia del perro ¿Te asustaste?

Brian: No.

Maestra: Pero yo lo que no sé ¿Adentro del departamento, qué... tenía un patio así, grande?

Brian: No, adentro no, afuera.

Maestra: En la calle.

Brian: No. Viste cuando entró mi mamá ahí, en el patio hay una puerta y del otro lado.

Maestra: En el departamento donde fuiste vos, tenía un patio grande, y en ese patio habia un perro.

La emisión líder con la que el niño inicia el intercambio constituye un resumen de la complicación del evento. En esta primera emisión, el niño centra el relato en su encuentro con el perro-Brian: Seño, ¿Sabes qué? El otro día yo fui a la casa del trabajo, un departamento chiquito con mi mamá y fui a abrir la puerta y salió un perro. No, primero estaba en el departamento tomando jugo, galletitas y mi mamá me dio... fue y había un perro que era muy grandote, que era grandote y fue al patio y salió el perro por la escalera, yo estaba en la escalera y el perro... salió-. En efecto, los sintagmas referidos al perro aparecen rematizados por el niño al final de la emisión-Brian:...salió un perro... había un perro... salió el perro-. Las repeticiones del niño-que era muy grandote, que era grandote... tenía una boca re grande-intensifican el impacto que el animal ha causado en él y transmiten implícitamente una evaluación de las impresiones del niño en esa situación.

Pero la maestra no atiende a estas pistas proporcionadas por el niño, interrumpe sus emisiones y, en lugar de brindarle un andamiaje para clarificar la secuencia de acciones referidas al encuentro con el perro, se centra en cuestiones tales como el trabajo de la mamá y las diferencias entre casas y departamentos - ¿Tu mamá trabaja en una casa?/ Es diferente en un departamento/¿Tu mamá limpia el departamento?/ ¿Y en el departamento tenía escalera para subir o...? ...¿ Había timbre, tenía timbre el departamento?/ ¿Adentro del departamento qué, tenía un patio así, grande?-. Al centrar por completo sus intervenciones en aspectos relativos a la orientación de la situación, aspectos que aparecen de un modo marcadamente periférico en el discurso del niño, la maestra construye otro texto paralelo que no respeta el tema propuesto por el niño-había un perro y casi me muerde-.

Sin embargo, el niño en distintos momentos del intercambio intenta recuperar su intención comunicativa -... y había un perro y casi me muerde/ Tenía una boca re grande- pero la maestra insiste en el desarrollo del tema de su interés: el trabajo de las madres $-{ }_{\text {¿Y }}$ tu mamá trabaja en una casa también?/ ¿Tu mamá limpia el departamento?/ Mirá que bueno, esas mamás que limpian las casas/ ¿Alguna otra mamá limpia, como la mamá de Brian?-. Cuando el niño, haciendo uso de recursos lingüísticos para atraer la atención de sus compañeros y crear una cierta tensión narrativa, afirma "tenía una boca re grande" la maestra debe aceptar que la historia del niño era otra $-M$ : seguimos con la historia del perro-. Aún reconociéndolo la maestra continúa elaborando su propio texto paralelo - Pero yo lo que no sé ¿Adentro del departamento, qué... tenía un patio así, grande?-.

De modo similar, en el siguiente intercambio la maestra pretende modificar la representación que una niña, Luana, tiene sobre la experiencia que relata. En este caso la emisión líder con la que Luana inicia un relato sobre su perra Akima expresa una evaluación en la que manifiesta sus sentimientos negativos en relación a su perra-La perrita Akima es mala-.

\section{(5) Luana (4: 6 años)}

Luana: La perrita Akima es mala.

Maestra: ¿Quién es Akima?

Luana: La hija de Bony.

Maestra: La perrita, la hija de Bony, la cachorrita.

Luana: Y la otra es de una gente que la vendió, que es buenita, es Luna y es buenita.

(1)

Maestra: Akima es mala y es la hija de Bony, y aparte ustedes compraron otro perro ...que se llama Luna, ¿Y de qué color es Luna?

Luana: Negra. 
Luana: (Akima) Ahora tiene dientes.

Maestra: ¿Y qué pasó con los dientes?

Luana: Lo mordió.

Maestra: ¿A quién mordió?

Luana: A Franco y a mí, a Edu, y a mi mamá y él. (Señala a un compañero).

Maestra: ¿A Lucas?... ¿Quiso morder a todos, pero jugando?

Luana: Sí y Luna tiene dientes pero es buenita.

...

Maestra: ¿Y a vos, quién te gusta más, Akima o Luna o Bony?

Luana: Luna.

Maestra: ¿Y si Akima está celosa, no lo pensaste? Luana: A mí me gusta Luna y Bony, Bony es buenita.

Maestra: Sí, ¿Y vos no pensaste que Akima puede estar celosa? Hay que hacerle un poco de caso también a Akima, hay que jugar también...

Maestra: Acabas de contar que tenés tres perros: Bony, Akima y...

\section{N: Luna}

Maestra: Y Luna. Pero vos queres a Luna y Bony, a Akima decís que no la querés porque se porta mal, porque te mordió a vos, a Lucas Alfaro, a Edu, tu papá y a tu mamá, pero a todos ellos muerde, pero jugando.

Luana: Si y tengo una perrita.

Maestra: ¡Otra más! ¿Cuatro perros tenés?

Luana: Sí, que se llama Negrita.

Maestra: ... ¿Y vos la querés también a Negrita?

Luana: A todas las perritas.

Maestra: A todas ¿A Akima también?

Luana: Sí.

Maestra: Ahora está bien.

Luana: Sabes qué

Maestra: A ver, vos me contaste que tenés cuatro perros: Bony, Akima, Luna y Negrita. Que Negrita es la más chiquita y después le sigue Bony, Akima y después Luna, ¿Si? Después me dijiste, ¿Qué más me contaste? Que Negrita era la más alta y la más bajita era Bony, ¿No? Y la más viejita era Negrita y que vos querés a todas por igual, las querés a todas.

Luana: Sí.

Maestra: Muy bien ¿Quién más tiene algo para contar?

Al comienzo del intercambio, si bien la maestra repite la afirmación de la niña que constituye su emisión líder - Akima es mala- desfocaliza esta in- formación central, ubicándola al comienzo de su emisión en una estructura coordinada con otra información periférica en el relato de la niña, estructura en la que se rematizan aspectos de la orientación que no constituyen el foco del relato de la niña $-M$ : Akima es mala y es la hija de Bony, y a parte ustedes compraron otro perro, perrito y perrita, perrita, que se llama Luna, ¿Y de qué color es Luna?-.

Luana defiende su intención comunicativa. En efecto, la niña continúa proporcionando comentarios evaluativos que tienen por foco aquellas características de los perros que son de su interés -Luna... es buenita... (en contraposición a "Akima es mala") -A mi me gusta Luna y Bony, Bony es buenita-. De esta manera, la niña retoma e intensifica los aspectos negativos de la perra al compararla con otros perros. Asimismo, alude a otra característica de la perra que se relaciona con la apreciación que tiene acerca de ella-tiene dientes/ muerde-.

En el último fragmento, las intervenciones de la maestra rematizan su propia interpretación evaluativa del comportamiento de la perra-Maestra: Está celosa/ Muerde, pero jugando. Hay que hacerle un poco de caso también a Akima-. La contradicción entre el sentido otorgado por la niña y el nuevo sentido que la maestra le imprime a los hechos se manifiesta lingüísticamente a través del uso del adversativo "pero": muerde "pero jugando".

Resulta interesante destacar que la maestra parece negociar este nuevo significado cuando por medio de preguntas le pide a la niña que confirme su interpretación - ¿La querés también a Negrita? ... ¿A Akima también?... ¿Las querés a todas por igual, las querés a todas?-. La relación asimétrica entre la maestra y la niña podría ser una de las razones por las cuales Luana asiente en distintas oportunidades a las intervenciones de la maestra que modifican el significado que ella quería transmitir-Maestra: ¿Y vos la querés también a Negrita? Luana: A todas las perritas-. La evaluación explícita de la maestra después de la confirmación de la niña del nuevo significado Ahora está bien- da cuenta del objetivo que la maestra había perseguido desde el principio: modificar la representación que la niña tenía del evento.

El tema del texto planteado por la niña-Akima es mala-así como las especificaciones remáticas posteriores de la niña-Luna es buenita/(Akima)Tiene dientes/Lo mordió/ Luna tiene dientes pero es buenital A mi me gusta Luna y Bony, Bony es buenita-son negadas por la evaluación final con la que la maestra termina el relato de la niña y contradicen el significado que la niña había querido transmi- 
tir-... Después me dijiste, qué más me contaste, que Negrita era la más alta y la más bajita era Bony, ¿No? Y la más viejita era Negrita y que vos querés a todas por igual, las querés a todas.

\section{Discusión}

El análisis presentado mostró diferencias en el grado en el que los textos producidos en las situaciones de interacción con la maestra respondían a la intención comunicativa inicial de los niños.

En algunas de las situaciones registradas se observó que las maestras ponían en juego estrategias variadas para recuperar el propósito de comunicación del niño, tal como podía inferirse de la emisión líder que constituía su turno de intervención inicial, y para colaborar con él en la elaboración de una narración canónicamente estructurada, que respetaba la perspectiva personal del niño en relación al evento relatado. En estos casos, las estrategias de colaboración de las maestras se apoyaban en la relación asimétrica que caracteriza su relación con los niños para "prestarles" sus representaciones más precisas tanto del conocimiento del mundo en general como de la estructura narrativa. De este modo, las maestras generaban una "zona de desarrollo potencial" en la que los niños podían, con este sostén estratégico, reconstruir su experiencia individual.

En otras situaciones, en cambio, se observó que las estrategias de interacción de las maestras se centraban en aspectos periféricos de los relatos infantiles. Las maestras se apoyaban en su relación asimétrica con los niños y se valían de recursos lingüísticos que permiten jerarquizar, focalizar y desfocalizar la información, para generar mayor fuerza comunicativa (Pardo, 1996) e imponer su argumento por sobre el del niño. En estos casos las maestras incluían las palabras del niño dentro de su propio razonamiento y, de ese modo, se apropiaban del relato infantil. El hecho de que en estas situaciones las maestras empleasen modos convencionales de cortesía (Brown \& Levinson, 1978) hace más difícil detectar el carácter de la interacción, aunque no por ello disminuye su impacto psicológico (Pardo, 1996).

Sin duda, diversas cuestiones pedagógicas, institucionales o personales más o menos conscientes subyacen a las acciones de las maestras que se "apropian" del relato infantil. Pero, independientemente de cuál sea la naturaleza de estas cuestiones, el resultado es el mismo, cuando se considera el hecho de que se reduce el espacio comunicativo del niño y se le quita la oportunidad de reelaborar su experiencia o, en palabras de Póveda (2001), de reflejar una visión del mundo y una construcción particular de la realidad.

¿Por qué a veces los adultos se alejan del significado y del sentido de la experiencia que los niños tienen interés en relatar? Como señalan Tessler (1986) y Nelson (1989), los niños y los adultos difieren en cuanto a los aspectos que consideran significativos de los eventos y en cuanto a la perspectiva que espontáneamente les imprimen al relatarlos.

El hecho de que, en algunas situaciones, el andamiaje de las maestras no recupere la perspectiva infantil acerca del evento, no puede atribuirse a que la maestra no comprenda el significado de las emisiones infantiles y no pueda inferir, por lo tanto, su intención comunicativa. En efecto, en las situaciones analizadas diversos indicadores mostraron que en todos los casos las maestras tenían cierta comprensión de lo que el niño quería transmitir. Tampoco puede esto atribuirse a impedimentos de los niños para manifestar su intención comunicativa. Por el contrario, en las situaciones analizadas abundan los ejemplos que manifiestan la insistencia de los niños por transmitir su punto de vista.

La relevancia de colaborar con los niños en la elaboración de narraciones que otorguen textura a su propia vida resulta evidente cuando se la considera en el marco de los resultados de las investigaciones de Haden, Ornstein, Eckerman y Didow (2001) y Fivush y Fromhoff(1988) realizadas en el medio familiar. Estas investigaciones muestran que los niños cuyas madres narrativizan su experiencia recuerdan los eventos pasados en mayor medida y de modo más organizado. Como sostiene Nelson (1996) el formato narrativo organiza la memoria de un modo coherente. Las conversaciones sobre los eventos pasados proporcionan a los niños un modelo sobre cómo organizar y recordar eventos específicos. Es por ello que el hablar sobre un evento permite preservarlo en la memoria. De este modo entre los 3 y los 3:6 años, a partir de la memoria episódica surge la memoria autobiográfica que permite la representación de los eventos significativos de la propia vida, contribuyendo a la conformación de la identidad. Por lo tanto, las diferencias en los estilos de relato de los padres y de otros adultos con los que los niños interactúan afectan no sólo la conformación de la memoria, sino también el desarrollo emocional en los años preescolares (Fivush \& Haden, 1997).

Asimismo, como se observó en las situaciones analizadas, los adultos espontáneamente contribuyen con evaluaciones e interpretaciones que 
explicitan las emociones de los niños. Tal como sugieren Fivush y Haden (1997), estas colaboraciones del adulto permiten que el niño comprenda, negocie y resuelva sentimientos de tristeza y temor en un contexto interactivo y aprenda a compartir y a evaluar sus emociones. En este proceso de representación y comunicación, afecto y cognición, aparecen íntimamente vinculados y resultan particularmente sensibles al impacto que sobre su desarrollo pueden tener las estrategias de interacción de los maestros en la escuela infantil.

\section{Referencias}

Brown, P. \& Levinson, S. (1978). Universals in language usage: Politeness phenomena. En E. Goody (Ed.), Questions and politeness. Cambridge papers in social antropology (pp. 56-289). Cambridge: Cambridge University Press.

Bruner, J. S. (1986). El habla del niño. Barcelona: Paidós. Bruner, J. S. (1988). Realidad mental y mundos posibles. Los actos de la imaginación que dan sentido a la experiencia. Barcelona: Gedisa.

Cazden, C. (1989). El discurso en el aula. El lenguaje de la enseñanza y el aprendizaje. Madrid: Paidós.

Firbas, J. (1992). Functional sentence perspective in written and spoken communication. Studies in English language. Cambridge: Cambridge University Press.

Fivush, R. \& Fromhoff, F. A. (1988). Style and structure in mother-child conversations about the past. Discourse Processes, 11, 337-355.

Fivush, R. \& Haden, C. A. (1997). Narrating and representing experience: Preeschooler's developing autobiographical accounts. En P. W. Van den Broek \& P. J. Bauer (Eds.), Developmental spans in event comprehension and representation (pp. 169-198). Hillsdale, NJ: Lawrence Erlbaum Associates.

Geertz, C. (1973). The interpretation of cultures: Selected essays. New York: Basic Books.

Gibaja, R. (1988). Acerca del debate metodológico en la investigación educacional. La educación. Revista Interamericana de Desarrollo Educativo (OEA), 103, 8194.

Glasser, B. \& Strauss, A. (1967). The discovery of grounded theory. Chicago: Aldine Publishing Company.

Green, M., Kantor, R. \& Rotger. (1991). Exploring the complexity of language and learning in classroom contexts. En B. Jonesy \& L. Idol (Eds.), Educational values and cognitive instruction: Implications for reform (pp. 331361). Hillsdale, NJ: Erlbawm.

Gumperz, J. (1984). Communicative competence revisited. En D. Schiffrin (Ed.), Meaning, form, and use in context: Linguistic applications (pp. 278-289). Washington: Georgetown University Press.

Haden, C. A., Ornstein, P. A., Eckerman, C. O. \& Didow, S. M. (2001). Mother-child conversational interactions as events unfold: Linkages to subsequent remembering. Child Development, 72(4), 1016-1031.

Labov, W. (1972). The transformation of experience in narrative discourse. En W. Labov (Ed.), Language in the inner city: Studies on the Black English vernacular (pp. 354-396). Filadelfia: University of Pennsylvania Press.
Mendez, L. \& Lacasa, P. (1997). El aula de una escuela de educación infantil: ¿Qué buscamos y qué hay en ella? Cultura y Educación, 8, 73-88.

Michaels, S. (1988). Presentaciones narrativas: Una preparación oral para la alfabetización con alumnos de primer curso. En J. Cook-Gumperz (Ed.), La construcción social de la alfabetización (pp. 109-136). Barcelona: Paidós.

Michaels, S. (1991). The dismantling of narrative. En A. McCabe \& C. Peterson (Eds.), Developing narrative structure (pp. 303-351). Hillsdale: Lawrence Erlbaum.

Miller, P. J. (1994). Narrative practices: Their role in socialization and self construction. En U. Neisser \& R. Fivush (Eds.), The remembered self: Construction and accuracy in the self narrative (pp. 158-179). New York: Cambridge University Press.

Miller, P. \& Sperry, L. (1987). Early talk about the past: The origins of conversational stories of personal experiences. Journal of Child Language, 15, 293-315.

Nelson, K. (1989). Monologue as representation of real life experience. En K. Nelson (Ed.), Narratives from the crib (pp. 27-32). Cambridge, MA: Harvard University Press.

Nelson, K. (1991). Events, narratives, memory: What develops? Ponencia presentada en The Minessota Symposium in Child Development, Emotion and Memory, Institute for Child Development, Institute of Minessota.

Nelson, K. (1996). Language in cognitive development. Cambridge: Cambridge University Press.

Ochs, E. \& Capps, L. (1996). Narrating the self. Annual Review of Anthropology, 25, 19-43.

Pardo, M. L. (1986). Hacia una redefinición de las nociones de tema y rema. De la oración al discurso. Filología, $X X I(1), 25-55$.

Pardo, M. L. (1996). Derecho y lingüistica. Cómo se juzga con palabras. Buenos Aires: Nueva Visión.

Póveda, D. (1999, agosto). Quico's story: An ethnopoetic analysis of a gipsy boy's narratives at school. Ponencia presentada en International Conference on Storytelling, St. Catharines, Canada.

Póveda, D. (2001, Spring Edition). Estructura e identidad en las narraciones escolares de un niño gitano. Sincronía: An E-Journal of Culture Studies. Recuperado desde http:// fuentes.csh.udg.mx/ccsh/Sincronía/index.htm.

Póveda, D. (2003). Paths to participation in classroom conversations. Linguistics and Education, 14(1), 69-98.

Póveda, D., Sebastián, E. \& Moreno, A. (2003). La ronda como evento para la constitución social del grupo en una clase de educación infantil. Infancia y Aprendizaje, 26(2), 131-146.

Preece, A. (1987). The range of narrative forms conversationally produced by young children. Child Language, 14(2), 353-373.

Reese, E., Haden, C. A. \& Fivush, R. (1993). Mother child conversations about the past: Relationships of style and memory over time. Cognitive Development, 8, 403-430.

Rosemberg, C. R. \& Borzone de Manrique, A. M. (1994). La interacción verbal en situaciones de lectura de cuentos en el aula. En R. Gibaja \& A. M. E. de Babini (Eds.), La educación en la Argentina: Trabajos actuales de investigación (pp. 165-204). Buenos Aires: La Colmena.

Rosemberg, C. R. \& Manrique, M. S. (2004). Relatos compartidos en la interacción entre la maestra y los niños en el jardín de infantes. Ponencia presentada en el II Coloquio Argentino de la IADA, International Association for Dialogue Analysis, Universidad de La Plata, Argentina. 
Rosemberg, C. R. \& Manrique, M. S. (2005, septiembre). Compartir experiencias personales en la escuela infantil. Un medio para reconstruir lingüisticamente la vida de los niños. Ponencia presentada en la IV Jornadas de Desarrollo Humano y Educación, Universidad de Alcalá de Henares, España.

Snow, C. E. (1983). Literacy and language: Relationships during the preschool years. Harvard Educational Review, 53(2), 165-189.

Strauss, A. \& Corbin, J. (1991). Basics of cualitative research. Grounded theory, procedures and technics. London: Sage Publications.

Fecha de recepción: Abril de 2006.

Fecha de aceptación: Junio de 2006.
Tessler, M. (1986). Mother-child talk in a museum: The socialization of a memory. Manuscrito no publicado, University of New York Graduate Center.

Tessler, M. \& Nelson, K. (1994). Making memories: The influence of joint encoding on later recall. Consciousness and cognition, 3, 307-326.

Valles, M. (2003). Técnicas cualitativas de investigación social. Madrid: Síntesis. 